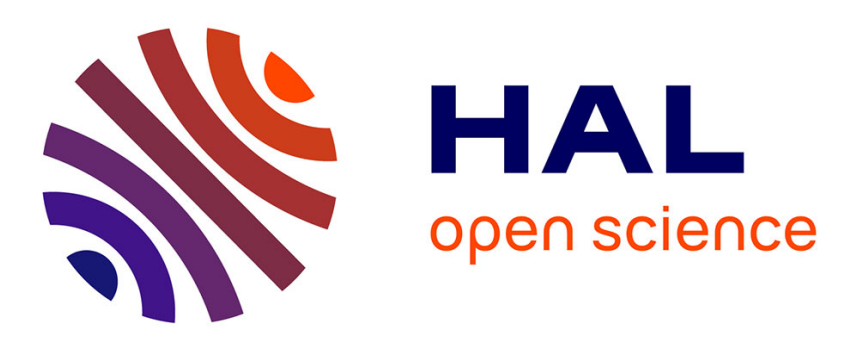

\title{
Quantitative Comparison of 3D Freehand Ultrasound and MRI Images of the Neonatal Brain
}

Martin Blanchard, Matthieu Martin, Philippe Quetin, Philippe Delachartre

\section{To cite this version:}

Martin Blanchard, Matthieu Martin, Philippe Quetin, Philippe Delachartre. Quantitative Comparison of 3D Freehand Ultrasound and MRI Images of the Neonatal Brain. 2020 IEEE International Ultrasonics Symposium (IUS), Sep 2020, Las Vegas (virtual), United States. 10.1109/IUS46767.2020.9251324 . hal-03148335

\section{HAL Id: hal-03148335 \\ https://hal.science/hal-03148335}

Submitted on 22 Feb 2021

HAL is a multi-disciplinary open access archive for the deposit and dissemination of scientific research documents, whether they are published or not. The documents may come from teaching and research institutions in France or abroad, or from public or private research centers.
L'archive ouverte pluridisciplinaire HAL, est destinée au dépôt et à la diffusion de documents scientifiques de niveau recherche, publiés ou non, émanant des établissements d'enseignement et de recherche français ou étrangers, des laboratoires publics ou privés. 


\section{Quantitative Comparison of 3D Freehand Ultrasound and MRI Images of the Neonatal Brain}

\author{
Martin Blanchard \\ CREATIS Laboratory, Lyon, France*
}

\author{
Matthieu Martin \\ CREATIS Laboratory, Lyon, France* \\ Philippe Delachartre \\ CREATIS Laboratory, Lyon, France* \\ delachartre@creatis.insa-lyon.fr
}

\author{
Philippe Quétin \\ $\mathrm{CH}$ Avignon, Avignon, France
}

*Univ Lyon, INSA-Lyon, Université Claude Bernard Lyon 1, UJM-Saint Etienne, CNRS, Inserm, CREATIS UMR
5220, U1206, F-69100, LYON, France.

\begin{abstract}
Premature neonates' care can be improved by a quantitative evaluation of brain structures. In current clinical practice, first-line diagnosis is done qualitatively with $2 \mathrm{D}$ cranial ultrasonography (cUS) images. These patients would benefit from an evolution to a quantitative analysis based on 3D cUS. A way to obtain high quality $3 D$ cUS images is to use a reconstruction algorithm based on the clinician acquisition motion. In this paper we assess the accuracy of such reconstruction by comparing brain structures between MRI and 3D renconstructed cUS. This comparison was performed based on manual contours, we obtained a mean Dice value of $0.72 \pm 0.05$ for thalami and ventricles and a mean volume error of $10 \pm 5.7 \%$. In addition, the contour interpolation method that we used significantly improved Dice and volume error compared to nearest neighbor approach.
\end{abstract}

Index Terms-3d ultrasound, MRI, preterm neonates, brain imaging, brain structure comparison.

\section{INTRODUCTION}

Preterm births represents $10 \%$ of mondial births (approximately 15 millions babies per year). These babies are prone to complications such as cerebral palsy or neurodevelopmental disorders [1]. To estimate theses risks a qualitative neurological imaging checkup is routinely performed after birth with 2D freehand cranial ultrasonography (cUS). Magnetic Resonance Imaging (MRI) is also performed to look for White Matter Injuries (WMI) in the case of great perterms or if cUS reveals some abnormalities.

Advanced MRI sequences have been developed in order to get biomarkers of neurodevelopment but this kind of MRI is done only in few research centers. In any case, MRI flaws are its accessibility and the fact that it can underestimate severe cavitary lesions, as well as moderate lesions such as Punctuate White Matter Lesions (PWML) [2].

Moreover, technological progress in ultrasound has enabled an easier description of hyperechoic moderate lesions of the white matter (WM) such as gliotic nodules (suggesting presence of PWML) and to propose a new classification of WMI [3].
Our current proposition leans on a 3D cUS obtained by reconstruction after a $2 \mathrm{D}$ freehand cUS acquisition which enables the evolution to $3 \mathrm{D}$ quantitative cUS.

To get cUS volume, a 3D reconstruction algorithm was implemented by [4]. This algorithm takes a pile of $2 \mathrm{D}$ cUS images as input and provide a 3D spatially coherent volume as output. As, this algorithm considered the doctor's movement as uniform during all the acquisition, there is a need to assess its efficiency by comparing the reconstructed brain structures with an acquisition from another modality. 3D cUS can also be achieved by tracking the probe like in [5].

Comparisons between MRI and 3D cUS have already been made in the past but rarely on cerebral imaging. To the best of our knowledge, only one study by Boucher et al. [6] compared cUS and MRI contours but only for ventricles.

In this paper, we developed a pipeline to measure the similarity between several cerebral structure in 3D cUS and MRI: cerebral ventricles, thalami and corpus callosum. To the best of our knowledge, this has never been done for thalami and corpus callosum. Registration between cUS and MRI has already been implemented by Fuerst et al. [7] but on whole volumes rather than contours of structures. Our study therefore differs because it does not try to fit MRI to US and vice versa but only to directly measure the difference between the cerebral structures in order to evaluate our reconstruction pipeline accuracy.

\section{MethoD}

\section{A. Data Acquisition and data segmentation}

cUS data were acquired on two patients through the anterior fontanel with an Acuson Siemens 9L4 multi-D matrix transducer. In order to get 3D Us images, the pile of 2D images from the cUS exam was processed with the 3D reconstruction pipeline proposed in [4]. On the same day, T1-weighted and T2-weighted MRI sequences were performed with a Philips 3T Ingenia. Pixel resolution of our images can be found on Table.1. 
Cerebral ventricles and thalami were manually annotated by a paediatrician. These structures are of major interest in our study because PWMLs are alongside the ventricles [8] and thalami volume has been proven to be negatively correlated with lesion load [9]. Because there were many slices in cUS (a mean of 235 slices per structure per volume, compared to 45 slices per structure per volume for MRI), the specialist only segments one among four images, which still took two 2 hours per structure on average. The cerebral structures were segmented on T1-weighed or T2-weighted MRI images, depending on which sequence they were visible.

The contours were used as inputs for the comparison pipeline proposed in this paper. An example of manual segmentation is given on Fig 1.

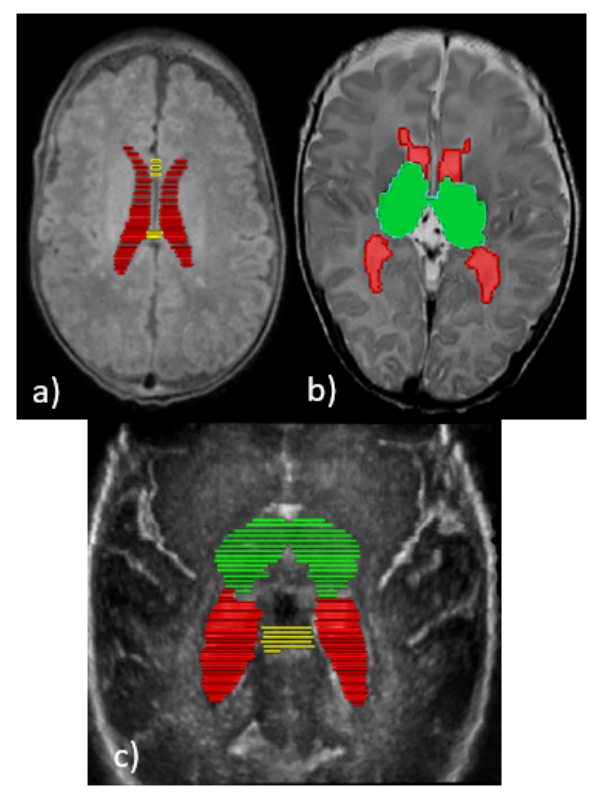

Fig. 1. Contours (Green: Thalami, Red: Ventricles, Yellow: Corpus Callosum) drawn on a) T1-weighted MRI, b) T2-weighted MRI, c) 3D cUS.

TABLE I

SUMMARY OF PIXEL RESOLUTION OF OUR SCANS (A: AXIAL ; S: SAGITTAL ; C: CORONAL).

\begin{tabular}{|c|c|c|c|}
\hline Patient & Exam & Resolution $(\mathbf{A x S x C )}(\mathbf{m m})$ & Slices Number $(\mathbf{A})$ \\
\hline$\# 1$ & T1 & $0.75 \times 0.84 \times 0.75$ & 319 \\
\hline$\# 1$ & T2 & $0.375 \times 2.5 \times 0.375$ & 85 \\
\hline$\# 2$ & T2 & $1.031 \times 1.1 \times 1.031$ & 43 \\
\hline All & cUS & $0.16 \times 0.16 \times 0.16$ & $\approx 600$ \\
\hline
\end{tabular}

\section{B. Comparison of the brain structures in US and MRI}

To compare brain structures between 3D cUS and MRI we developped a pipeline which enable to obtain complete segmentation in the US volume and which to register the contours drawn in US and MRI images. The obtaining of full segmentation in 3D cUS volume is summarized in Fig.2. The steps of this pipeline, which will be described more precisely in the next section, are:

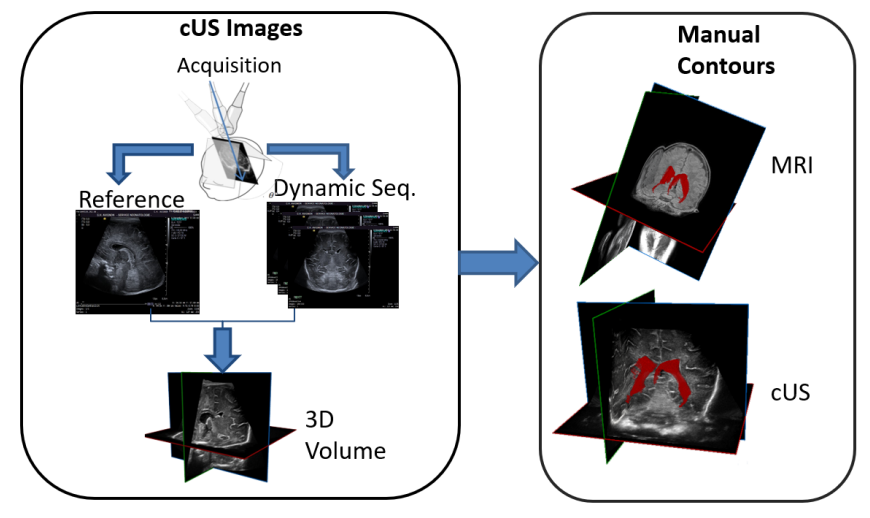

Fig. 2. Summary of our reconstruction pipeline.

1) Interpolation: As the contours were not performed on all 3D cUS images, an interpolation was realised in order to get fully segmented volumes.

2) Cropping: To optimise the computational efficiency of the pipeline, the cUS volumes, which consisted of approximately 350 millions voxels, were cropped to a region of interest.

3) Upscaling: In order to have the same resolution in US and MRI volumes, MRI images were upscaled to cUS resolution.

4) Rigid registration: Both volume were then rigidly registered to each other. We chose not to use elastic registration in order to preserve the cerebral structures shape and their true dimensions.

5) Dice and volume calculation: The volume of both structures and the Dice between them was finally calculated to estimate their similarity.

\section{Pipeline step description}

\section{Interpolation}

In order to interpolate contours in all slices located between two manually segmented images, interpolated distance maps to the border of the contours were used.

Let $\Omega_{i}$ be the set of points representing the manual segmentation of a given structure on a slice $S_{i}$. Each point $x_{\text {map }}$ of the signed distance map $m_{i}$ associated to $S_{i}$ was calculated as follow :

$$
x_{\text {map }}= \begin{cases}d(x, \partial \Omega) & \text { if } x \in \Omega \\ -d(x, \partial \Omega) & \text { if } x \in \bar{\Omega}\end{cases}
$$

where $\partial \Omega$ defines the boundary of $\Omega, \bar{\Omega}$ the complement of $\Omega$ and $d$ is defined such that :

$$
d(x, \partial \Omega)=\min _{y \in \partial \Omega} d(x, y)
$$

Given an empty slice $S_{i+k}$ between two segmented slices $S_{i}$ and $S_{i+e}$, the distance map $m_{i+k}, k \in[|0, e|]$, was calculated with equation 3 as follows:

$$
m_{i+k}=m_{i} \times \frac{e-k}{e}+m_{i+e} \times \frac{k}{e} .
$$


A threshold was finally applied to the distance maps, all negative values were put to 0 and all positive values to 1 .

\section{Registration}

Given our two volumes respectively described by $f(x, y, z)$ and $g(x, y, z)$, we estimate the transformation $\Psi(x, y, z, q)$ with $q=\left(t_{x}, t_{y}, t_{z}, \Theta_{x}, \Theta_{y}, \Theta_{z}\right)$, parameters of the transformation - by minimizing the following square error given by equation 4:

$$
H(q)=\sum_{x, y, z}[f(x, y, z)-g(\Psi(x, y, z, q))]^{2} .
$$

This loss was used because of the binary nature of the volume. $H$ was minimised using gradient descent and $q$ was initialized at $q_{0}=(0,0,0,0,0,0)$. At each step $k$, the gradient $\nabla H\left(q_{k}\right)$ was computed, if it was under a threshold $\epsilon=10^{-6}$, the minimisation was stopped, otherwise $q_{k+1}$ was calculated as follows:

$$
q_{k+1}=q_{k}-\alpha \nabla H\left(q_{k}\right) .
$$

with $\alpha=0.001$ the learning rate. The maximum number of iterations was set to $M=150$.

\section{Dice and volume calculation}

To evaluate the accuracy of our pipeline, the difference between the segmentation in cUs and MRI volumes was measured with Dice, which is the gold-standard for measuring the similarities between 2 contours. Given two sets $E$ and $F$ representing segmentation, Dice was calculated as follows:

$$
\operatorname{Dice}(E, F)=2 \times \frac{\operatorname{card}(E \cap F)}{\operatorname{card}(E)+\operatorname{card}(F)} .
$$

In addition, we calculated the structure volumes and the relative volume error between two structures:

$$
S E\left(V_{M R I}, V_{C U S}\right)=\frac{\left|V_{M R I}-V_{C U S}\right|}{V_{M R I}} .
$$

Where |.| is the absolute value and $V_{M R I}$ and $V_{c U S}$ respectively correspond to the volume measured in MRI and cUS volumes. These volumes were calculated by multiplying the pixel volume by the number of pixel contained in the structure.

The pipeline was implemented with Python, using simpleITK [10].

\section{RESULTS AND DISCUSSION}

The similarity between cUS and MRI contours was measured on three cerebral structures: ventricles, thalami and corpus callosum. Table II presents the Dice and Volume error results for two different patients, Patient \#1 with T1 and T2weighted MRI and Patient \#2 with only T2-weighted MRI.
TABLE II

Dice and Volume ERror (LV: LATERAL Ventricles ; TH: Thalami ; CC: CORpus CALlosum)

\begin{tabular}{|c|c|c|c|c|}
\hline Patient & Structure & MRI sequence & Dice & Volume Error \\
\hline$\# 1$ & LV & T1 & $\mathbf{0 . 7 2}$ & $3 \%$ \\
\hline$\# 1$ & TH & T2 & $\mathbf{0 . 8 5}$ & $18 \%$ \\
\hline$\# 1$ & CC & T1 & 0.44 & $>20 \%$ \\
\hline$\# 1$ & LV & T2 & $\mathbf{0 . 6 9}$ & $8 \%$ \\
\hline$\# 2$ & LV & T2 & 0.64 & $5 \%$ \\
\hline$\# 2$ & TH & T2 & $\mathbf{0 . 7 0}$ & $20 \%$ \\
\hline
\end{tabular}

A mean Dice of $0.72 \pm 0.05$ was obtained for thalami and ventricles, which is encouraging considering the low resolution of most of the MRI scans. A Dice of 0.44 was found in the case of the corpus callosum which was under our expectations but can be explained by an acceleration during the cUS acquisition which resulted in a misrepresent corpus callosum (Fig. 3). This shows the need for a probe tracking during acquisition.
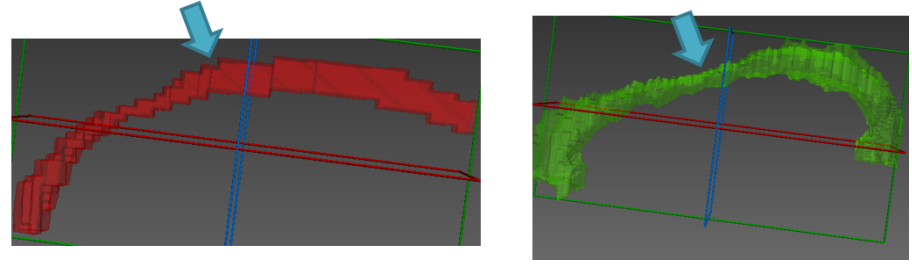

Fig. 3. Comparison of corpus callosum from Patient \#1 (Red: T1-weighted MRI ; Green: 3D cUS). Arrows point to the body of the corpus callosum to highlight the shape difference between cUS and MRI.

The Ventricle volume error was $5.3 \pm 1.7 \%$ which is similar to the values reported by [6] and the mean Dice for this structure was $0.68 \pm 0.03$. Visual results can be seen on Fig. 4.

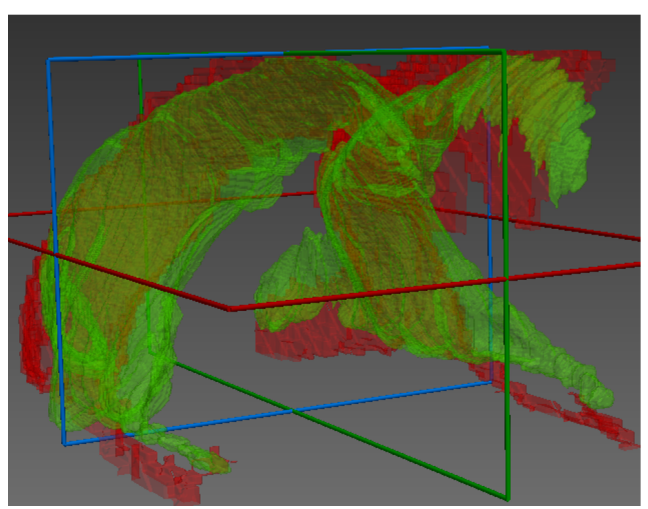

Fig. 4. Comparison of ventricles from Patient \#1 (Red: T1-weighted MRI ; Green: cUS). Dice: 0.72 ; Volume Error: $3 \%$.

Even if thalami volume error was very high, Dice was still very satisfying $(0.8 \pm 0.05)$. It is also important to note that thalami segmentation is very difficult and therefore can result in a higher volume error. Visual results can be seen on Fig. 5. 


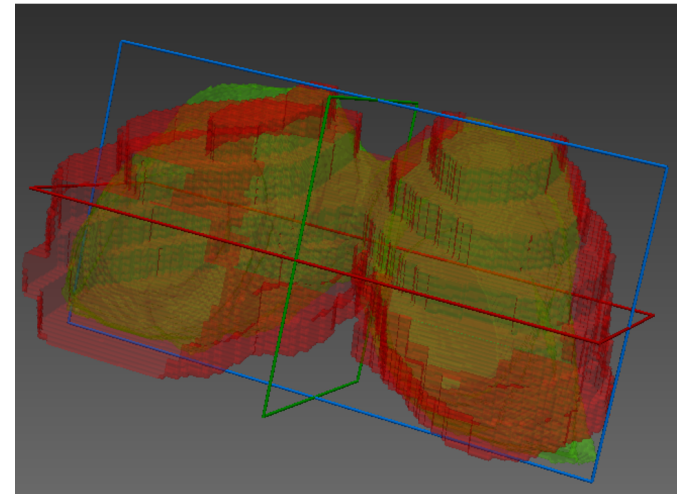

Fig. 5. Comparison of thalami from Patient \#1 (Red: T1-weighted MRI ; Green: cUS). Dice: 0.85 ; Volume Error: $18 \%$.

In order to assess the accuracy of the proposed distance map method to interpolate the segmentation in cUS volumes, we tried the nearest neighbor interpolation as an alternative method. Comparison for the LV contours of Patient \#1 are given in Table III.

TABLE III

DICE AND VOLUME ERROR RESULTS FOR TWO DIFFERENT TYPES OF INTERPOLATIONS ON LV OF PATIENT \#1

\begin{tabular}{|c|c|c|}
\hline Interpolation & Dice & Volume Error \\
\hline Nearest Neighbor & 0.63 & $8 \%$ \\
\hline Distance Maps & $\mathbf{0 . 7 2}$ & $3 \%$ \\
\hline
\end{tabular}

These results show that our interpolation method provides a better volume approximation and a better Dice. This means that it was better at reconstructing the contours in the empty slices.

Apart from thalami which presents great Dice results, performances of our pipeline were limited by the resolution and the number of slices in MRI volumes. Nevertheless, a decent Dice of 0.72 as well as a small volume error of $3 \%$ were obtained in the case of LV for the MRI with the highest number of slices and the most isotropic voxels (T1-weighted MRI of Patient \#1).

\section{CONCLUSION}

In this study, we developed a pipeline to evaluate the similarities between 3D cUS and MRI contours of structures such as ventricles, corpus callosum and thalami. To the best of our knowledge this had never been done in the case of corpus callosum and thalami. The best Dice was obtained by the thalami and LV gave us a good Dice and the lowest volume error. These results are very encouraging for the use of 3D cUS as a high-resolution alternative imaging modality to cerebral MRI in the case of preterm babies.

In a future work, MRI acquisitions with a higher resolution will be performed and our database size will be increased. Moreover, we also plan to improve the 3D US reconstruction algorithm accuracy by tracking the probe speed and angle.

\section{ACKNOWLEDGMENT}

This work was performed within the framework of the LABEX CELYA (ANR-10-LABX-0060) and PRIMES (ANR11-LABX-0063) of Universite de Lyon, within the program "Investissements d'Avenir" (ANR-11-IDEX-0007) operated by the French National Research Agency (ANR). We would like to thank Dr. Borhane Slama, the innovation and clinical research commission president of $\mathrm{CH}$ Avignon, and the $\mathrm{CH}$ Avignon direction for their support to this research.

\section{REFERENCES}

[1] Arpino, C., Compagnone, E., Montanaro, M.L. et al. Preterm birth and neurodevelopmental outcome: a review. Childs Nerv Syst 26, 1139-1149 (2010). https://doi.org/10.1007/s00381-010-1125-y.

[2] Martines-Biarge M. et al. "MRI Based Preterm White Matter Injury Classification: The Importance of Sequential Imaging in Determining Severity of Injury". PLoS ONE 11(6): e0156245. doi:10.1371/journal.pone.0156245

[3] Agut T. et al. "Preterm white matter injury: ultrasound diagnosis and classification". Pediatric Research (2020) 87:37-49; https://doi.org/10.1038/s41390-020-0781-1

[4] Martin M., B. Sciolla, M. Sdika, X. Wang, P. Quetin and P. Delachartre "Automatic Segmentation of the Cerebral Ventricle in Neonates Using Deep Learning with 3D Reconstructed Freehand Ultrasound Imaging," 2018 IEEE International Ultrasonics Symposium (IUS), Kobe, 2018, pp. 1-4, doi: 10.1109/ULTSYM.2018.8580214.

[5] Herickhoff CD et al. "Low-cost Volumetric Ultrasound by Augmentation of 2D Systems: Design and Prototype". In: Ultrasonic Imaging 40(1) (2018), p. 35-48.

[6] Boucher M.A. et al. Computer-aided lateral ventricular and brain volume measurements in 3D ultrasound for assessing growth trajectories in newborns and neonates. Phys Med Biol 63, 225-237 (2018). https://doi.org/10.1088/1361-6560/aaea85.

[7] Fuerst B. et al. Automatic ultrasound-MRI registration for neurosurgery using the 2D and 3D LC2 Metric. MIA 18(8), 1312-1319 (2014). https://doi.org/10.1016/j.media.2014.04.008.

[8] Raybaud C. et al. "The Premature Brain: Developmental and Lesional Anatomy". Neuroradiology 55 (S2): 23-40 (2013).

[9] Tusor N., Benders, M.J., Counsell, S.J. et al. "Punctate White Matter Lesions Associated With Altered Brain Development And Adverse Motor Outcome In Preterm Infants". Sci Rep 7, 13250 (2017). https://doi.org/10.1038/s41598-017-13753-X

[10] R. Beare et al., "Image Segmentation, Registration and Characterization in R with SimpleITK", J Stat Softw, 86(8), doi: 10.18637/jss.v086.i08, 2018. 